\section{A review article on artificial intelligence}

\author{
Nagendraswamy C* and Amogh Salis
}

JSS College of Nursing, Mysuru, Karnataka, India

\begin{abstract}
Artificial intelligence $(\mathrm{Al})$ is the emulation of human intelligence in computers that have been trained to think and behave like humans. The word may also refer to any computer that exhibits human-like characteristics like learning and problem-solving. Artificial intelligence is intelligence demonstrated by machines, as opposed to natural intelligence, which involves consciousness and emotionality and is demonstrated by humans and animals [1].
\end{abstract}

More Information

*Address for Correspondence:

Nagendraswamy C, Assistant Lecturer, JSS

College of Nursing, Mysuru, Karnataka, India,

Email: saruswati28@gmail.com

Submitted: April 22, 2021

Approved: May 18, 2021

Published: May 19, 2021

How to cite this article: Nagendraswamy C, Salis A. A review article on artificial intelligence. Ann Biomed Sci Eng. 2021; 5: 013-014.

DOI: 10.29328/journal.abse.1001012

Copyright: ๑ 2021 Nagendraswamy C, et al. This is an open access article distributed under the Creative Commons Attribution License, which permits unrestricted use, distribution, and reproduction in any medium, provided the original work is properly cited.

Keywords: Intelligence; Computers

\section{Introduction}

Artificial intelligence (AI) refers to intelligence D) Check for updates (6) OPEn Access demonstrated by computers as opposed to natural intelligence demonstrated by humans and animals, which includes consciousness and emotionality. The acronym chosen often reveals the distinction between the first and second grades. Artificial General Itelligence (AGI) is the term used to describe'strong' AI [2].

\section{Types of Al [3]}

1. Machines that respond. Reactive robots are the most basic kind of robot.

2. Memory is limited. As the name implies, a limited memory machine is capable of retaining certain information gleaned from previous events or records.

3. Self-awareness...

4. Theory of Mind [4].

\section{Purposes}

- Al's mission is to create software that can reason and justify based on feedback.

- AI will allow humans to communicate with software in a human-like manner.

- provide decision support for specific tasks [5].

\section{Examples of AI}

* Cheques can now be deposited from the comfort of our own home.

* AI is capable of deciphering handwriting,
* online check processing is possible.

* Artificial intelligence can also be used to detect fraud by looking at users' credit card spending habits.

* Alexa.

* SIRI.

\section{The world smartest AI}

The Lincoln Laboratory Supercomputing Center's (LLSC) new TX-GAIA (Green AI Accelerator) computing system has been named the world's most efficient artificial intelligence supercomputer [6].

\section{Applications of AI}

D-Commerce and Artificial Intelligence.

> AI in Navigation, GPS technology, according to MIT research, can provide users with reliable, timely, and comprehensive information to improve protection.

$>\mathrm{AI}$ in Robotics.

$>$ AI in Human Resource.

$>\mathrm{AI}$ in Healthcare.

$>\mathrm{AI}$ in Agriculture.

$>\mathrm{AI}$ in Gaming. 


\section{Growth drivers of $\mathrm{AI}$ in health care}

> The rising amount of healthcare data and increasing complexity of datasets driving the need for AI.

> The intensifying need to reduce soaring healthcare costs.

> Improving computing capacity and decreasing hardware costs.

$>$ The number of AI-enabled medical devices are the major factors driving the artificial intelligence in healthcare market development [7].

\section{Challenges in AI}

* Computing Capacity Most developers are turned off by the amount of power these power-hungry algorithms consume.

* The Bias Issue.

* Limited Knowledge.

* Human-level.

* Data Privacy and Security.

* The Trust Deficit.

* Scarcity of data [8].

\section{Conclusion}

The use of machine-learning algorithms and software, or artificial intelligence (AI), to simulate human cognition in the study, presentation, and comprehension of complex medical and health-care data is referred to as artificial intelligence in healthcare. Refers to the replication of human intelligence in computers that have been programmed to think and act like humans. The phrase can also refer to any machine that demonstrates human-like characteristics like learning and problem-solving.

\section{References}

1. Frankenfield J. Reviewed by Gordon Scott. 2021. https://www investopedia.com

2. Poole, Mackworth \& Goebel. Which provides the version that is used in this article. These authors use the term "computational intelligence" as a synonym for artificial intelligence. 1998.

3. Reynoso R. 4 Main Types of Artificial Intelligence. 2019. g2.com

4. Artificial Intelligence - What it is and why it matters. SAS India. 2021.

5. Foy K. Lincoln Laboratory. 2019. news.mit.edu

6. https://executive-education-online.mit.edu/

7. Artificial Intelligence in Healthcare Market. 2020.

8. Vadapalli P. Top 7 Challenges in Artificial Intelligence in 2021. 